\title{
Germany clambers aboard translational research bandwagon
}

$\mathrm{I}$ t's almost as if a slow wave is overtaking the medical research enterprise in the western world.

Scant weeks after the National Institutes of Health in the United States indicated that it will reform its programming to speed the delivery of new drugs and therapies (www.cmaj.ca/cgi/doi /10.1503/cmaj.109-3777), the government of Germany has announced a new health program aimed at shortening the time it takes for the results of basic and clinical research to become part of regular medical care.

"We want to get new treatments to the patients as fast as possible," Annette Schavan, Germany's minister of research, told a press conference at which she and Health Minister Philipp Rösler sketched the new program's priorities over the course of the next eight years.

The program focuses on studying common diseases such as cancer, diabetes and Alzheimer disease, as well as cardiovascular and infectious diseases. To that end, the government will spend $€ 5.5$ billion on health research between 2011-2014. That will include spending on personalized medicine, prevention, nutrition and health care delivery research.

The "centrepiece of the program," Schavan said, will be the creation of four more national health research centres. She hopes those will make translation - the application of new medical knowledge in the clinic or hospital faster and more effective. It is also hoped the centres will make more efficient use of funds, pool resources to enable large studies and attract topflight international talent.

Two national centres, the German Centre for Neurodegenerative Diseases and the National Centre for Diabetes Research, have already opened. They'll be joined this year by others dedicated to research on cardiovascular diseases, infectious diseases, lung diseases and cancer.

It constitutes a seismic shift in the structure and organization of German

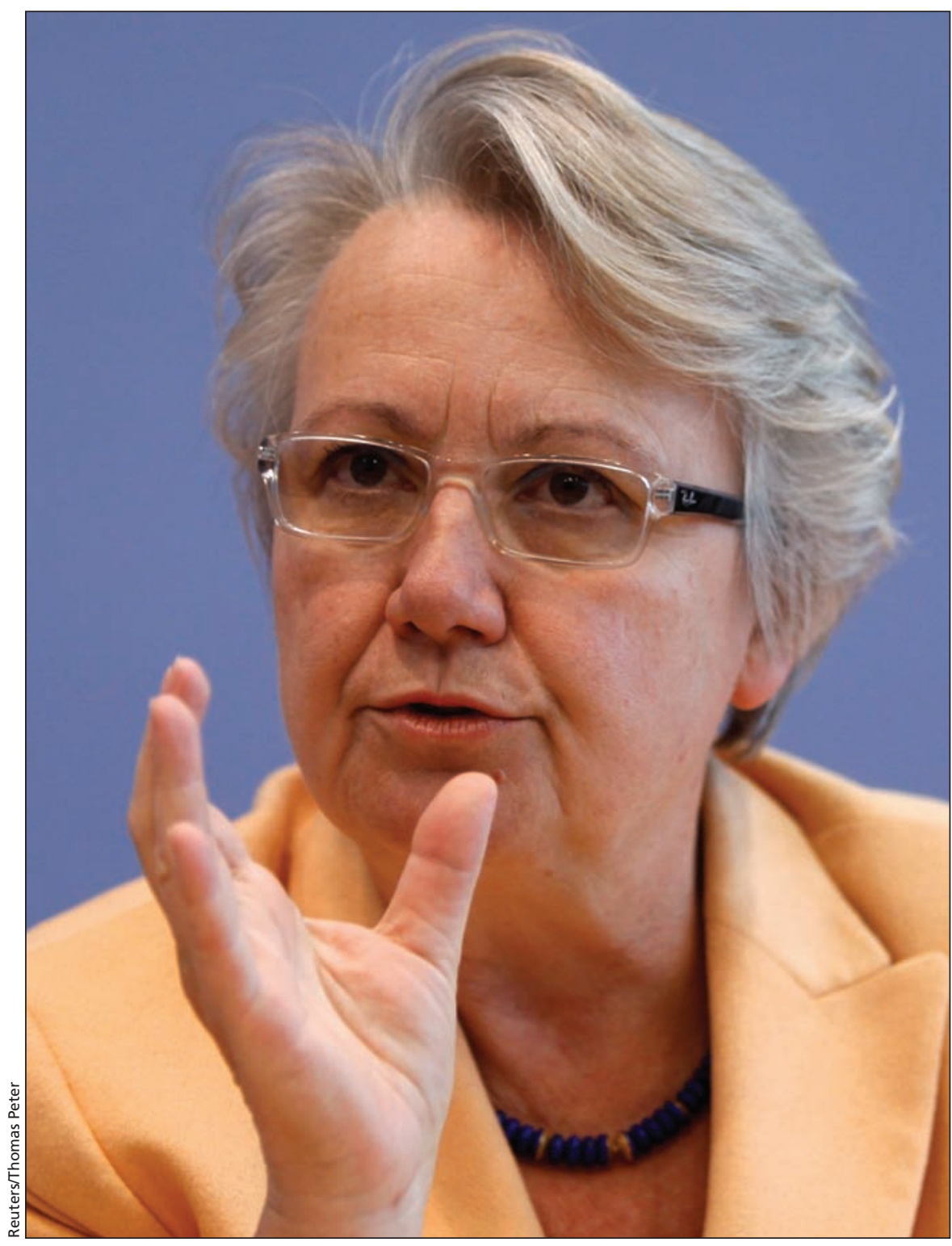

"We want to get new treatments to the patients as fast as possible," Annette Schavan, Germany's minister of research, told a press conference while announcing that her government will spend $€ 5.5$ billion on health research between 2011-2014.

health research. Traditionally, biomedical research has been done either by universities and university clinics, or by independent research institutes typically funded by societies.

The new centres, though, will be collaborative ventures between universities and research institutes, Schavan said. "We want to bring the best people in a field together."
There has been some grumbling among a few researchers in response to the government initiative, largely around which researchers were chosen, or not chosen, to be part of a national centre.

In the case of the National Centre for Infectious Diseases, for example, several have expressed surprise that neither tuberculosis researcher Stefan Kaufmann 
nor nosocomial infection expert Dr. Petra Gastmeier were selected. "Some researchers, that one would have liked to be in there, did not make it," says Dr. Dieter Bitter-Suermann, president of the German Medical Faculty Association and head of research at the Hannover Medical School, which is part of the infectious diseases institute.

Others query the priorities of the infectious diseases institute. "Viral diseases, even exotic ones are very well represented in the centre. But sepsis, which kills 144 people every day, is not," says Dr. Konrad Reinhart, director of the department of anesthesiology and intensive care medicine at the University Hospital of the Friedrich-Schiller University of Jena and coordinator of Competence Network Sepsis, a network of experts conducting clinical and experimental sepsis research.

"Connecting the best researchers in a field is a good idea," he says. "But if important diseases such as sepsis are not included, then that will be a big problem in the long run," says Reinhart, who along with Gastmeier penned a letter to the government criticizing the omission of sepsis and hospital- acquired infections in the infectious diseases institute's research program.

Other contentious issues involve the funding, structure and organization of the institutes. As the German government is prohibited from directly funding universities, the $€ 500$ million that is ticketed for the six centres over the next four years will be funnelled through the Helmholtz Society, a union of 16 research centres with a budget of $€ 3$ billion annually, $90 \%$ of which comes from the federal government.

Critics charge that the arrangement gives the Helmholtz Society too much power. "In the first two centres, the Helmholtz Society is in effect like the spider in the middle of the net," BitterSuermann says. Some fear that amounts to institutes under the wing of the Helmholtz Society being able to essentially buy access to patients and patient data, which would be invaluable in recruiting participants for clinical trials.

Because of such criticism, three of the new centres - for cardiovascular, infectious and lung diseases - will have structures in which universities will be given more input into how available monies will be spent.

It means that the six national health research centres will fall into two camps: those centrally organized and under the wing of the Helmholtz Society, and three in which universities will have more influence. "It will be very interesting to see how these two groups do," BitterSuermann says.

Many predict glowing outcomes and impacts. "This is a huge change and a huge chance," says Dr. Otmar Wiestler, head of the German Cancer Research Centre in Heidelberg, which will lead the new centre for translational cancer research.

But not all the centres will be equally effective immediately, he adds. "At the moment, it takes 15 years to go from a medical discovery to it being part of patient care. We need to reduce that to 5 to 10 years."

The pooling of talent and resources should aid in that, says Dr. Simone Fulda, professor for experimental tumour research at the University of Frankfurt and a member of the translational cancer centre. "We will only be able to solve the big challenges in medicine if we get all the experts to work together." - Kai Kupferschmidt, Berlin, Germany

CMAJ 2011. DOI:10.1503/cmaj.109-3795 